\title{
Static Magnetic Field Effects on Impaired Peripheral Vasomotion in Conscious Rats
}

\author{
Shenzhi Xu, ${ }^{1,2}$ Hideyuki Okano, ${ }^{2,3}$ Masaaki Nakajima, ${ }^{4}$ Naoya Hatano, ${ }^{1}$ \\ Naohide Tomita, ${ }^{1}$ and Yoshito Ikada ${ }^{5}$ \\ ${ }^{1}$ Department of Mechanical Engineering and Science, Graduate School of Engineering, Kyoto University, Kyoto 606-8502, Japan \\ ${ }^{2}$ Research Section for Magnetics, Product Development Department, Development Division, PIP Company, Osaka 540-0011, Japan \\ ${ }^{3}$ Research Center for Frontier Medical Engineering, Chiba University, Chiba 263-8522, Japan \\ ${ }^{4}$ Department of Physical Therapy, School of Health Science and Social Welfare, Kibi International University, \\ Okayama 716-8508, Japan \\ ${ }^{5}$ Department of Medical Engineering, Nara Medical University, Nara 634-8522, Japan
}

Correspondence should be addressed to Hideyuki Okano; hideyukiokano@aol.com

Received 9 August 2013; Accepted 7 November 2013

Academic Editor: Ke Ren

Copyright (C) 2013 Shenzhi Xu et al. This is an open access article distributed under the Creative Commons Attribution License, which permits unrestricted use, distribution, and reproduction in any medium, provided the original work is properly cited.

\begin{abstract}
We investigated the SMF effects on hemodynamics in the caudal artery-ligated rat as an in vivo ischemia model using noninvasive near-infrared spectroscopy (NIRS) combined with power spectral analysis by fast Fourier transform. Male Wistar rats in the growth stage (10 weeks old) were randomly assigned into four groups: (i) intact and nonoperated cage control $(n=20)$; (ii) ligated alone ( $n=20)$; (iii) ligated and implanted with a nonmagnetized rod (sham magnet; $n=22)$; and (vi) ligated and implanted with a magnetized rod $(n=22)$. After caudal artery ligation, a magnetized or unmagnetized rod (maximum magnetic flux density of $160 \mathrm{mT}$ ) was implanted transcortically into the middle diaphysis of the fifth caudal vertebra. During the experimental period of 7 weeks, NIRS measurements were performed in 3-, 5- , and 7-week sessions and the vasomotion amplitude and frequency were analyzed by fast Fourier transform. Exposure for 3-7 weeks to the SMF significantly contracted the increased vasomotion amplitude in the ischemic area. These results suggest that SMF may have a regulatory effect on rhythmic vasomotion in the ischemic area by smoothing the vasomotion amplitude in the early stage of the wound healing process.
\end{abstract}

\section{Introduction}

Increasing evidence of the importance of moderate-intensity magnetic field influence on in vivo physiological function has led to the consideration of medical applications of this knowledge [1-25]. For example, magnetic field therapy using moderate-intensity static magnetic fields (SMF; $1 \mathrm{mT}-1 \mathrm{~T}$ ) could be useful for circulatory diseases, including ischemic pain, inflammation, and hypertension, primarily due to the modulation of blood flow and/or blood pressure. In our previous studies, we have shown that subchronic exposure (3-12 weeks) to SMF $(180 \mathrm{mT})$ accelerates bone recovery from operative invasion and ischemia induced by artery ligation [23, 24], or ovariectomy-induced osteoporosis [25], as indicated by the increased amount of bone mineral density (BMD). These studies suggested that SMF improved BMD probably due to recovery of microcirculation from bone ischemia. With regard to the effects of SMF on spectral properties of microcirculatory vasomotion, acute exposure (30$40 \mathrm{~min})$ to SMF (30-223 $\mathrm{mT}$ ) has been investigated for rats [14] and humans [20]. However, for prolonged exposure to SMF, there is no direct evidence. Focused on the subchronic exposure (3-7 weeks) to SMF (160 mT), the present study was undertaken to evaluate the effects of SMF on the vasomotion amplitude and frequency by fast Fourier transform.

\section{Methods}

2.1. Animals. Male Wistar rats of 10 weeks old and weighing 310-360 g were used for this study (Charles River Laboratories Japan Inc., Kyoto, Japan). All animal experiments were 


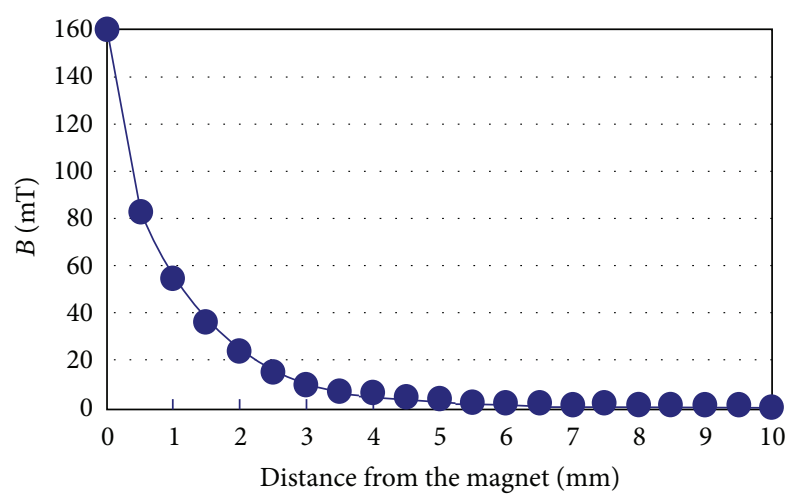

(a)

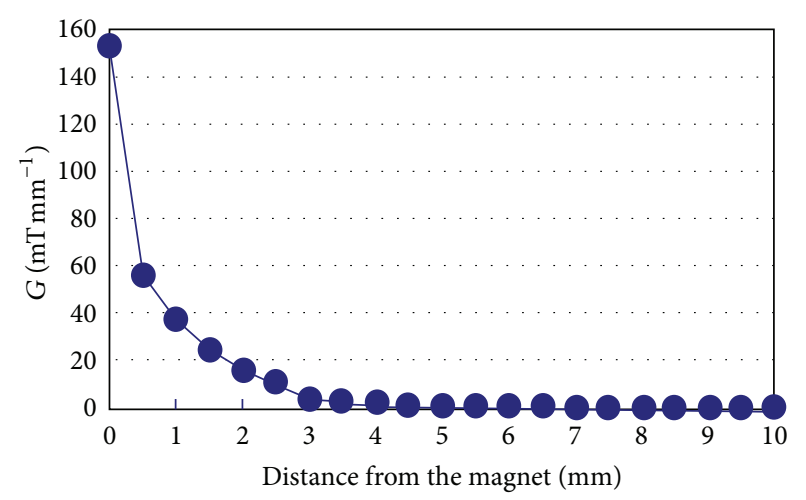

(b)

FIgURE 1: (a) Spatial distribution of the magnetic flux density values. (b) Spatial distribution of the magnetic gradient values.

approved by the Kyoto University Animal Research Committee, and all the experimental procedures were conducted in accordance with the guiding principles of the Guide for the Care and Use of Laboratory Animals published by the National Institutes of Health (NIH Publication no. 85-23, revised in 1996).

2.2. Static Magnetic Fields. A tapered rod was prepared from a samarium-cobalt alloy being designed as an elongated, narrow truncated cone (large diameter, $1.5 \mathrm{~mm}$; small diameter, $1.1 \mathrm{~mm}$; length, $6 \mathrm{~mm}$; and weight, $61 \mathrm{mg}$ ), with or without magnetization of the rod. The entire surface was homogeneously coated with polyimide to prevent the release of metal ions from the alloys into the body. Every piece of magnet had a maximum magnetic flux density $\left(B_{\max }\right)$ of $160 \mathrm{mT}$ and a maximum magnetic gradient $\left(G_{\max }\right)$ of $154 \mathrm{mT} \mathrm{mm}^{-1}$ (Figure 1). Both the $B_{\max }$ and $G_{\max }$ values were observed at the center of the large diameter surface (South pole) of the rod end of the magnet. The second highest values $(B, 131 \mathrm{mT}$; $G, 116 \mathrm{mT} \mathrm{mm}^{-1}$ ) were observed at the center of the small diameter surface (North pole), and the higher values were localized on both polar ends of the magnet perpendicular to the axis. The magnetic flux density was measured from the magnetic rods using a gaussmeter (Model 4048, Hall probe A-4048-002, Bell Technologies). All rods were sterilized by immersing in $0.5 \%$ dilute Hibitane solution for $30 \mathrm{~min}$ before implantation.

2.3. Experimental Procedure. After general intraperitoneal (i.p.) anesthesia with medetomidine $\left(180 \mu \mathrm{gkg}^{-1}\right)$ and midazolam $\left(1.25 \mathrm{mgkg}^{-1}\right)$ in rats as described in our previous report [25], the dorsal caudal skin just over the fifth caudal vertebra was incised. The caudal artery was ligated with a suture and amputated at the fifth caudal vertebra. Caudal circulation just after the ligation was mainly supported by collateral vessels. The rod was vertically implanted into the fifth caudal vertebra in a similar manner as described in our previous reports $[23,24]$. Briefly, for implantation, a $2 \mathrm{~cm}$ lateral skin incision was made, and, thereafter, the fifth caudal

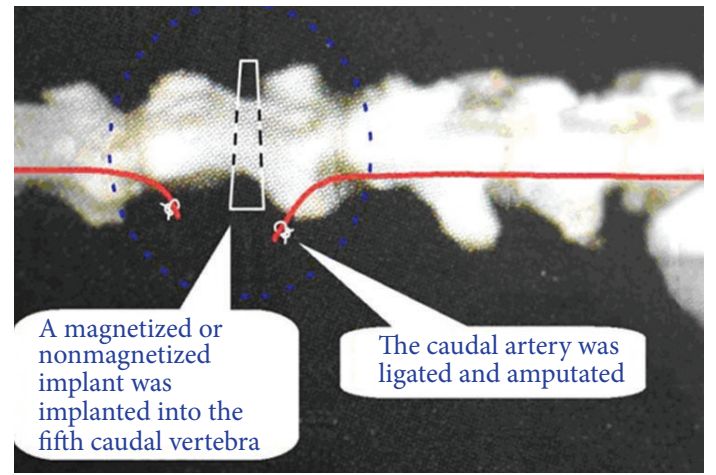

FIGURE 2: The caudal artery was ligated with a suture and amputated at the fifth caudal vertebra. Caudal circulation just after the ligation was mainly supported by collateral vessels. The rod was vertically implanted into the fifth caudal vertebra.

vertebra was exposed by blunt dissection of th caudal muscle. The periosteum was incised and pushed aside to drill a hole in the middle diaphysis of the fifth caudal vertebra from the lateral cortex to the medical cortex. The drill size was matched to that of the rod. A rod was implanted transcortically into the hole, applying a load of $500 \mathrm{~g}$ for $30 \mathrm{~s}$ using a digital force gauge. Thereafter, three layers of muscle, subcutaneous connective tissue, and skin were sutured. Spatial distribution of the magnetic flux density values in the caudal muscle (target tissue) was $160 \mathrm{mT}$ or less $\left(B_{\max }=160 \mathrm{mT}\right)$.

All rats were randomly assigned to one of four groups: (i) intact and nonoperated cage control (C); (ii) ligated alone (L); (iii) ligated and implanted with a nonmagnetized rod (sham magnet, $\mathrm{L}+\mathrm{S}$ ); and (vi) ligated and implanted with a magnetized $\operatorname{rod}(\mathrm{L}+\mathrm{M})$. The sham magnet without magnetization was prepared for the rod used with the same size and materials as those of the magnet. The sham magnet was implanted in the same position of another rat to compare the effect with the magnet (Figure 2).

After the operation, two rats were housed together in one cage $\left(\mathrm{LWH}, 340 \times 240 \times 170 \mathrm{~mm}^{3}\right)$. All animals had free 
access to water and standard pellet food and were kept under controlled lighting conditions (12:12 h light-dark cycle) at room temperature and relative humidity $\left(25 \pm 1^{\circ} \mathrm{C}\right.$ and $55 \pm$ $5 \%)$.

2.4. Measurements of Blood Circulation. After a 3-week healing period, peripheral blood circulation in the caudal muscle was estimated as tissue blood oxygen contents using noninvasive near-infrared spectroscopy (NIRS, BOM-LITRW, Omega wave, Tokyo, Japan; during the healing period up to 3 weeks, the measurements were not carried out due to the suture-based intervention). The NIRS measurements of peripheral circulation in the ligated portion of caudal artery were performed in prone position under conscious condition (without anesthesia) in 3-, 5-, and 7-week sessions. The measurements in ligated alone group (L group) and control group (C group) were also conducted in the same manner. Thereafter, the vasomotion amplitude and frequency of the data were analyzed by Fourier transform. For the measurement, each rat was placed into an acrylic holder for a loose restraint. Prior to measurements, each rat was acclimated to the holder and tail cuff for at least $10 \mathrm{~min}$ without anesthesia. The measurement duration was $5 \mathrm{~min}$.

NIRS is based on the weak absorption of near-infrared light by biological tissues and, in particular, in this measurement system, tissue levels of blood oxygen contents, that is, oxygenated hemoglobin $(\mathrm{OxyHb})$, deoxygenated hemoglobin (DeoxyHb), and total hemoglobin (Total $\mathrm{Hb}$ ), can be determined using three laser diodes $(780,810$, and $830 \mathrm{~nm}$ ) according to the Beer-Lambert law. The absorption coefficient of hemoglobin at each wavelength is based on the data reported by Matcher et al. [26]. In addition, the value of tissue blood oxygen saturation $\left(\mathrm{StO}_{2}\right)$ can be calculated as:

$$
\mathrm{StO}_{2}=\frac{\mathrm{OxyHb}}{\text { Total } \mathrm{Hb}} \times 100(\%)
$$

It has been reported that NIRS parameters reflected the oxygenation consumption $\left(\mathrm{VO}_{2}\right)$ in the local muscle by Kawaguchi et al. [27]. For taking the measurements, the laser transmitting probe and receiving detector were fixed on both lateral sides of the tail skin surface at the healed surgical incision site with a tail cuff without compression. Because the tail diameter was less than $10 \mathrm{~mm}$, the fixed distance between the probe and detector was $10 \mathrm{~mm}$, with a resulting penetration depth of $10 \mathrm{~mm}$ from the tail skin surface. The data were inputted into a personal computer at sampling frequency of $60 \mathrm{~Hz}$. The analytical data were calculated one frame per one sec.

Statistical analysis was performed using one-way ANOVA followed by Bonferroni-Dunn post hoc test (StatView 5.0, SAS Institute Inc., USA) for each group of measurements $(P<0.05)$. All of the data were expressed as mean \pm S.D.

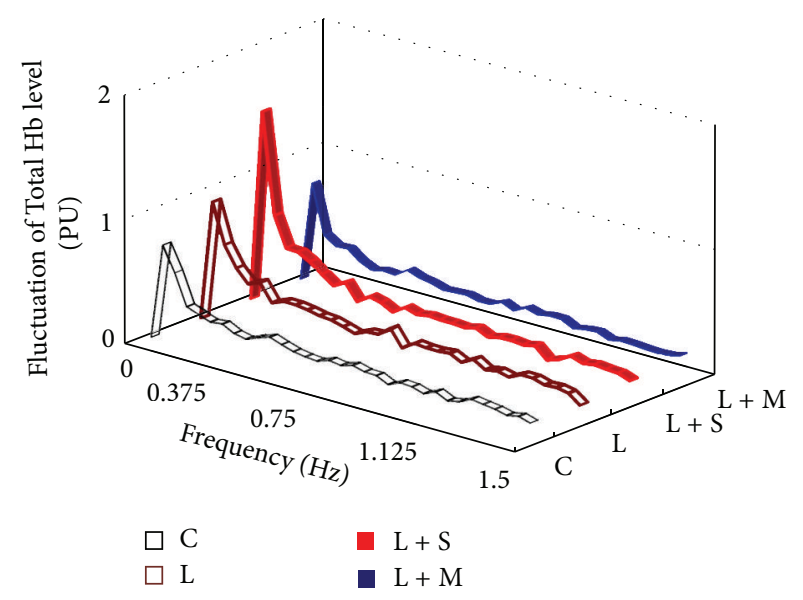

FIGURE 3: Relationship between vasomotion amplitude and frequency of the mean Total $\mathrm{Hb}$ level change in a 3-week exposure period. $\mathrm{C}, n=20 ; \mathrm{L}, n=20 ; \mathrm{L}+\mathrm{S}, n=22 ; \mathrm{L}+\mathrm{M}, n=22$. PU: perfusion unit.

\section{Results}

3.1. The Average Values of the Blood Circulation Parameters. In terms of the average values of the four blood circulation parameters $\left(\mathrm{OxyHb}\right.$, DeoxyHb, Total $\mathrm{Hb}$, and $\mathrm{StO}_{2}$ ) in blood circulation in the caudal muscle, there was no significant difference between the groups in 3-, 5-, and 7-week sessions (data not shown).

3.2. The Average Values of the Vasomotion Amplitude and Frequency. Fourier transform analysis indicated that the average values of vasomotion amplitude in four blood circulation parameters peaked at the frequency of $0.05 \mathrm{~Hz}$, for example, the amplitude of Total $\mathrm{Hb}$ level change in a 3-week session (Figure 3). Therefore, at the frequency of $0.05 \mathrm{~Hz}$, the average values of vasomotion amplitude were statistically compared between the groups. The average value of vasomotion amplitude for Total $\mathrm{Hb}$ in the $\mathrm{L}+\mathrm{S}$ group was significantly larger than those for the $\mathrm{C}$ group, $\mathrm{L}$ group, and $\mathrm{L}+\mathrm{M}$ group in a 3-week session $(P<0.05$; Figure 4$)$. Moreover, there was also no significant difference between the groups in 5- and 7week sessions (data not shown). For other parameters, that is, DeoxyHb and $\mathrm{StO}_{2}$ values, there was no significant difference between the groups during the experimental period.

\section{Discussion}

Previous researches have shown that acute exposure (30$40 \mathrm{~min})$ to SMF $(30-223 \mathrm{mT})$ increased skin vasomotion amplitude $[14,20]$. In resting skin blood flow in healthy young men, Yan et al. [20] found that SMF exposure at $B_{\max }$ of $223 \mathrm{mT}$ for $30 \mathrm{~min}$ induced a significant increase in vasomotion amplitude, mainly reflecting the intrinsic myogenic and endothelial-related metabolic activities, by placing a magnet to the center of the middle finger prominence and, after removal of the SMF, the vasomotion amplitude vanished gradually. In an animal (rat) model, Li et al. [14] 


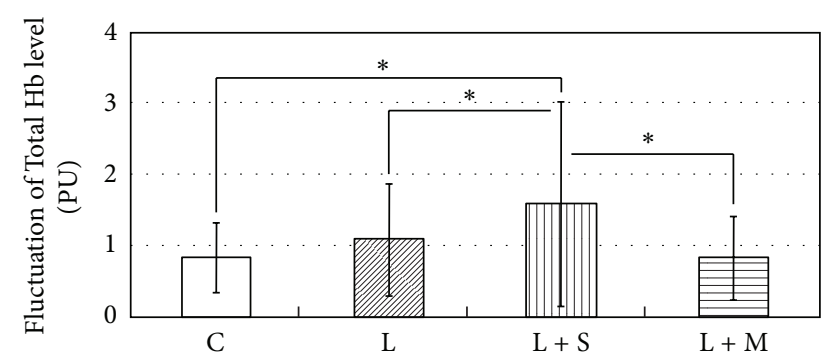

Figure 4: Fluctuation of Total $\mathrm{Hb}$ level at the frequency of $0.05 \mathrm{~Hz} 3$ weeks after ligated and implanted operation. C, $n=20 ; \mathrm{L}, n=20 ; \mathrm{L}+$ $\mathrm{S}, n=22 ; \mathrm{L}+\mathrm{M}, n=22$. Mean \pm S.D. PU: perfusion unit; ${ }^{*} P<0.05$.

reported significant enhancement of vasomotion amplitude, mainly reflecting the endothelial-related metabolic activity $(0.01-0.05 \mathrm{~Hz})$ in the skin stressed by pressure loading over the trochanter area upon exposure to an SMF at $B_{\max }$ of $30 \mathrm{mT}$ for $40 \mathrm{~min}$. In their study, prolonged surface loading caused significant reduction of the endothelial-related metabolic activity and increased the myogenic activity, that is, induced a higher vascular tone in tissues that had been stressed as compared with the unstressed ones [14]. In contrast, SMF significantly increased the endothelial-dependent vasodilation and subsequently increased blood flow in the stressed skin [14]. The modulating effect of SMF on the vasomotion amplitude might be related to the vascular tone modified by prolonged compressive loading [14].

In contrast to the aforementioned previous studies $[14,20]$, we focused on examining the subchronic effects of moderate-intensity inhomogeneous SMF on peripheral hemodynamics. The present study indicated that SMF exposure for 3 weeks seems to have the tendency to modulate the vasomotion amplitude at $0.05 \mathrm{~Hz}$ in the range of endothelialrelated metabolic activity for rats, and contract the increased vasomotion amplitude in the ischemic area, but did not induce significant change in any one of these parameters during the SMF exposure period of 3-7 weeks investigated. These results suggest that SMF may have a regulatory effect on rhythmic vasomotion in the ischemic area by smoothing the vasomotion amplitude, mainly reflecting the endothelialrelated metabolic activity, in the early stage of the wound healing process. The physiological implication is that the smoothing or buffering of the vasomotion amplitude may play a key role on inherent hemodynamic control mechanisms for rhythmic vasomotion and endothelial-dependent vasodilation.

Other reports have shown that an SMF of strong intensity higher than a few Tesla has bioeffects [28-41]. For instance, an SMF of strong intensity with an extremely high magnetic gradient $\left(B_{\max }\right.$ of $8 \mathrm{~T}$ and $G_{\max }$ of $\left.400 \mathrm{~T}^{2} / \mathrm{m}\right)$ could induce some bioeffects on paramagnetic hemoglobin by magnetic attraction in a high gradient or diamagnetic hemoglobin by magnetic repulsion in a high gradient, retarding the mean blood velocity in peripheral circulation, partly due to the asymmetric distribution of red blood cells (RBC) with different magnetic susceptibilities and magnetically induced movement of diamagnetic water vapor at the skin surface, which may lead to a skin temperature decrease [36]. Moreover, it has been shown that RBC rotate and orient so that the concave surface is aligned parallel to strong uniform SMF due to magnetic torque [29]. Concerning the SMF effects on blood viscosity, different results were obtained, depending on the exposure conditions [34, 40, 41]. Haik et al. [34] reported an increase in viscosity of blood flowing parallel to inhomogeneous strong SMF at $B_{\max }$ of 3,5 , and $10 \mathrm{~T}$. Yamamoto et al. [40] also indicated an increased viscosity of both fully oxygenated and fully deoxygenated blood and greater increase in blood viscosity of deoxygenated blood relative to that of oxygenated blood in $1.5 \mathrm{~T}$ homogeneous SMF exposure for $36 \mathrm{~min}$. In contrast, Tao and Huang [41] demonstrated that acute exposure to $1.33 \mathrm{~T}$ homogeneous SMF reduced blood viscosity when it was applied parallel to the flow direction (for an exposure duration of $1 \mathrm{~min}$, short chains of RBC were formed; for an exposure duration of $12 \mathrm{~min}$, long cluster chains of RBC were formed. This process could enable the RBC to pass through the blood vessels in a more streamlined fashion, thereby reducing the blood viscosity) [41]. These observations suggested that both inhomogeneous and homogeneous high intensity SMF (Tesla level) may modulate in vivo hemodynamics. However, the underlying mechanisms and physiological consequences have not yet been fully understood.

Because our applied SMF at $B_{\max }$ of $160 \mathrm{mT}$ was much lower in the magnetic force compared with the SMF of several Tesla, different plausible mechanisms might exist between them, such as through a $\mathrm{Ca}^{2+}$-calmodulin-regulated NOcGMP signaling pathway. Takeshige and Sato [1] suggested a mechanism of SMF action for the promotion of hemodynamic responses in which an SMF at $B_{\max }$ of $130 \mathrm{mT}$ might inhibit acetylcholinesterase (AChE). Recovery of circulation is assumed to be partly due to the enhanced release of acetylcholine (ACh) by the SMF exposure, activating the cholinergic vasodilator nerve endings innervated to the muscle artery [1]. The inhibitory effect of SMF on AChE was also observed in the magnetic flux density of $0.8 \mathrm{mT}$ or more [42]. In addition, it is also suggested that an SMF at $B_{\max }$ of $5.5 \mathrm{mT}$ should have a potential to counteract the action of a nitric oxide synthase (NOS) inhibitor L-NAME, presumably via increased endogenous ACh release $[43,44]$. The increased (upregulated) effect of a $120 \mu \mathrm{T}$ SMF on endothelial nitric oxide synthase (eNOS) expression was also confirmed in human umbilical vein endothelial cells (HUVEC) [45].

In our study, SMF did not cause any significant change in DeoxyHb during the experimental period. In contrast, more recently, Muehsam et al. [44] found that exposure for 10$30 \mathrm{~min}$ to $\mathrm{SMF}$ of $B_{\max } 186 \mathrm{mT}$ resulted in more rapid $\mathrm{Hb}$ deoxygenation, using a reducing agent dithiothreitol in an in vitro cell-free preparation. Thus, in their study, SMF significantly increased the rate of $\mathrm{Hb}$ deoxygenation occurring several minutes to several hours after the end of SMF exposure [46]. The observation that SMF pretreatment of $\mathrm{Hb}$ alone or of the deoxygenation solution itself failed to yield a significant effect suggests that SMF exposure acted upon the interaction of $\mathrm{Hb}$ with the deoxygenation solution [46]. With regard to the mechanism, they speculated that these 
SMF modalities modified protein/solvation structure in a manner that altered the energy required for deoxygenation [46]. They further speculated that the mT-range SMF could induce the action of the Lorentz force on charges bound at the protein/water interface based on the Lorentz-Langevin model for weak magnetic field bioeffects $[47,48]$. This model suggested that weak exogenous AC/DC magnetic fields can act on an ion/ligand bound in a molecular cleft based upon the assumption that the receptor molecule is able to detect the Larmor trajectory of an ion or ligand within the binding site. To date, however, there is insufficient direct experimental evidence pertaining to this model. Further studies are required to better understand the mechanisms of SMF bioeffects on hemodynamic function. Spectral analysis would be useful to examine these effects in more detail with a view of hemodynamics.

\section{Conclusion}

Exposure for 3-7 weeks to the SMF at $B_{\max }$ of $160 \mathrm{mT}$ and $G_{\max }$ of $154 \mathrm{mT} \mathrm{mm}^{-1}$ significantly contracted the increased vasomotion amplitude in the ischemic area. It seems possible that SMF may have a regulatory effect on rhythmic vasomotion in the ischemic area by smoothing the vasomotion amplitude in the early stage of the wound healing process.

\section{References}

[1] C. Takeshige and M. Sato, "Comparisons of pain relief mechanisms between needling to the muscle, static magnetic field, external qigong and needling to the acupuncture point," Acupuncture and Electro-Therapeutics Research, vol. 21, no. 2, pp. 119-131, 1996.

[2] C. Ohkubo and S. Xu, "Acute effects of static magnetic fields on cutaneous microcirculation in rabbits," In Vivo, vol. 11, no. 3, pp. 221-225, 1997.

[3] S. Xu, H. Okano, and C. Ohkubo, "Subchronic effects of static magnetic fields on cutaneous microcirculation in rabbits," In Vivo, vol. 12, no. 4, pp. 383-389, 1998.

[4] H. Okano, J. Gmitrov, and C. Ohkubo, "Biphasic effects of static magnetic fields on cutaneous microcirculation in rabbits," Bioelectromagnetics, vol. 20, no. 3, pp. 161-171, 1999.

[5] S. Xu, H. Okano, and C. Ohkubo, "Acute effects of whole-body exposure to static magnetic fields and $50-\mathrm{Hz}$ electromagnetic fields on muscle microcirculation in anesthetized mice," Bioelectrochemistry, vol. 53, no. 1, pp. 127-135, 2001.

[6] C. E. Morris and T. C. Skalak, "Static magnetic fields alter arteriolar tone in vivo," Bioelectromagnetics, vol. 26, no. 1, pp. $1-9,2005$.

[7] H. N. Mayrovitz and E. E. Groseclose, "Effects of a static magnetic field of either polarity on skin microcirculation," Microvascular Research, vol. 69, no. 1-2, pp. 24-27, 2005.

[8] C. E. Morris and T. C. Skalak, "Chronic static magnetic field exposure alters microvessel enlargement resulting from surgical intervention," Journal of Applied Physiology, vol. 103, no. 2, pp. 629-636, 2007.

[9] J. C. McKay, F. S. Prato, and A. W. Thomas, "A literature review: the effects of magnetic field exposure on blood flow and blood vessels in the microvasculature," Bioelectromagnetics, vol. 28, no. 2, pp. 81-98, 2007.
[10] C. E. Morris and T. C. Skalak, "Acute exposure to a moderate strength static magnetic field reduces edema formation in rats," American Journal of Physiology, vol. 294, no. 1, pp. H50-H57, 2008.

[11] G. Brix, S. Strieth, D. Strelczyk et al., "Static magnetic fields affect capillary flow of red blood cells in striated skin muscle," Microcirculation, vol. 15, no. 1, pp. 15-26, 2008.

[12] S. Strieth, D. Strelczyk, M. E. Eichhorn et al., "Static magnetic fields induce blood flow decrease and platelet adherence in tumor microvessels," Cancer Biology \& Therapy, vol. 7, no. 6, pp. 814-819, 2008.

[13] D. Strelczyk, M. E. Eichhorn, S. Luedemann, G. Brix, M. Dellian, and A. Berghaus, "Static magnetic fields impair angiogenesis and growth of solid tumors in vivo," Cancer Biology and Therapy, vol. 8, no. 18, pp. 1756-1762, 2009.

[14] Z. Li, E. W. C. Tam, A. F. T. Mak, and R. Y. C. Lau, "Wavelet analysis of the effects of static magnetic field on skin blood flowmotion: investigation using an in vivo rat model," In Vivo, vol. 21, no. 1, pp. 61-68, 2007.

[15] M. S. Markov, "Magnetic field therapy: a review," Electromagnetic Biology and Medicine, vol. 26, no. 1, pp. 1-23, 2007.

[16] M. S. Markov, "Therapeutic application of static magnetic fields," Environmentalist, vol. 27, no. 4, pp. 457-463, 2007.

[17] Z. Wang, P. Yang, H. Xu, A. Qian, L. Hu, and P. Shang, "Inhibitory effects of a gradient static magnetic fieldon normal angiogenesis," Bioelectromagnetics, vol. 30, no. 6, pp. 446-453, 2009.

[18] M. S. Markov, "Angiogenesis, magnetic fields and 'window effects," Cardiology, vol. 117, no. 1, pp. 54-56, 2010.

[19] S. Kim, Y. A. Chung, C. U. Lee, J. Chae, R. Juh, and J. Jeong, "Target-specific rCBF changes induced by 0.3 -T static magnetic field exposure on the brain," Brain Research, vol. 1317, pp. 211217, 2010.

[20] Y. Yan, G. Shen, K. Xie et al., "Wavelet analysis of acute effects of static magnetic field on resting skin blood flow at the nail wall in young men," Microvascular Research, vol. 82, no. 3, pp. 277-283, 2011.

[21] C. Ohkubo and H. Okano, "Clinical aspects of static magnetic field effects on circulatory system," Environmentalist, vol. 31, no. 2, pp. 97-106, 2011.

[22] S. Ueno and H. Okano, "Static, low frequency and pulsed magnetic fields in biological systems," in Electromagnetic Fields in Biological Systems, J. C. Lin, Ed., pp. 115-196, CRC Press, Boca Raton, Fla, USA, 2011.

[23] S. Xu, N. Tomita, R. Ohata, Q. Yan, and Y. Ikada, "Static magnetic field effects on bone formation of rats with an ischemic bone model," Bio-Medical Materials and Engineering, vol. 11, no. 3, pp. 257-263, 2001.

[24] S. Xu, N. Tomita, K. Ikeuchi, and Y. Ikada, "Recovery of small-sized blood vessels in ischemic bone under static magnetic field," Evidence-Based Complementary and Alternative Medicine, vol. 4, no. 1, pp. 59-63, 2007.

[25] S. Xu, H. Okano, N. Tomita, and Y. Ikada, "Recovery effects of a $180 \mathrm{mT}$ static magnetic field on bone mineral density of osteoporotic lumbar vertebrae in ovariectomized rats," Evidence-Based Complementary and Alternative Medicine, vol. 2011, Article ID 620984, 8 pages, 2011.

[26] S. J. Matcher, C. E. Elwell, C. E. Cooper, M. Cope, and D. T. Delpy, "Performance comparison of several published tissue near-infrared spectroscopy algorithms," Analytical Biochemistry, vol. 227, no. 1, pp. 54-68, 1995. 
[27] K. Kawaguchi, M. Tabusadani, K. Sekikawa, Y. Hayashi, and K. Onari, "Do the kinetics of peripheral muscle oxygenation reflect systematic oxygen intake?" European Journal of Applied Physiology, vol. 84, no. 1-2, pp. 158-161, 2001.

[28] T. S. Tenforde, C. T. Gaffey, B. R. Moyer, and T. F. Budinger, "Cardiovascular alterations in Macaca monkeys exposed to stationary magnetic fields: experimental observations and theoretical analysis," Bioelectromagnetics, vol. 4, no. 1, pp. 1-9, 1983.

[29] T. Higashi, A. Yamagishi, T. Takeuchi et al., "Orientation of erythrocytes in a strong static magnetic field," Blood, vol. 82, no. 4, pp. 1328-1334, 1993.

[30] T. Shiga, M. Okazaki, A. Seiyama, and N. Maeda, "Paramagnetic attraction of erythrocyte flow due to an inhomogeneous magnetic field," Bioelectrochemistry and Bioenergetics C, vol. 30, pp. 181-188, 1993.

[31] T. Higashi, A. Yamagishi, T. Takeuchi, and M. Date, "Effects of static magnetic fields of erythrocyte rheology," Bioelectrochemistry and Bioenergetics, vol. 36, no. 2, pp. 101-108, 1995.

[32] J. M. Denegre, J. M. Valles Jr., K. Lin, W. B. Jordan, and K. L. Mowry, "Cleavage planes in frog eggs are altered by strong magnetic fields," Proceedings of the National Academy of Sciences of the United States of America, vol. 95, no. 25, pp. 14729-14732, 1998.

[33] D. J. Snyder, J. W. Jahng, J. C. Smith, and T. A. Houpt, "c-Fos induction in visceral and vestibular nuclei of the rat brain stem by a 9.4 T magnetic field," NeuroReport, vol. 11, no. 12, pp. 26812685, 2000.

[34] Y. Haik, V. Pai, and C. Chen, "Apparent viscosity of human blood in a high static magnetic field," Journal of Magnetism and Magnetic Materials, vol. 225, no. 1-2, pp. 180-186, 2001.

[35] H. Kotani, H. Kawaguchi, T. Shimoaka et al., "Strong static magnetic field stimulates bone formation to a definite orientation in vitro and in vivo," Journal of Bone and Mineral Research, vol. 17, no. 10, pp. 1814-1821, 2002.

[36] S. Ichioka, M. Minegishi, M. Iwasaka et al., "Skin temperature changes induced by strong static magnetic field exposure," Bioelectromagnetics, vol. 24, no. 6, pp. 380-386, 2003.

[37] M. Zborowski, G. R. Ostera, L. R. Moore, S. Milliron, J. J. Chalmers, and A. N. Schechter, "Red blood cell magnetophoresis," Biophysical Journal, vol. 84, no. 4, pp. 2638-2645, 2003.

[38] O. Valiron, L. Peris, G. Rikken et al., "Cellular disorders induced by high magnetic fields," Journal of Magnetic Resonance Imaging, vol. 22, no. 3, pp. 334-340, 2005.

[39] C. H. Hsieh, M. C. Lee, J. J. Tsai-Wu et al., "Deleterious effects of MRI on chondrocytes," Osteoarthritis and Cartilage, vol. 16, no. 3, pp. 343-351, 2008.

[40] T. Yamamoto, Y. Nagayama, and M. Tamura, "A bloodoxygenation-dependent increase in blood viscosity due to a static magnetic field," Physics in Medicine and Biology, vol. 49, no. 14, pp. 3267-3277, 2004.

[41] R. Tao and K. Huang, "Reducing blood viscosity with magnetic fields," Physical Review E, vol. 84, no. 1, Article ID 011905, 2011.

[42] S. Ravera, B. Bianco, C. Cugnoli et al., "Sinusoidal ELF magnetic fields affect acetylcholinesterase activity in cerebellum synaptosomal membranes," Bioelectromagnetics, vol. 31, no. 4, pp. 270276, 2010.

[43] H. Okano and C. Ohkubo, "Modulatory effects of static magnetic fields on blood pressure in rabbits," Bioelectromagnetics, vol. 22, no. 6, pp. 408-418, 2001.

[44] H. Okano and C. Ohkubo, "Anti-pressor effects of whole body exposure to static magnetic field on pharmacologically induced hypertension in conscious rabbits," Bioelectromagnetics, vol. 24, no. 2, pp. 139-147, 2003.

[45] C. F. Martino, H. Perea, U. Hopfner, V. L. Ferguson, and E. Wintermantel, "Effects of weak static magnetic fields on endothelial cells," Bioelectromagnetics, vol.31, no. 4, pp. 296-301, 2010.

[46] D. Muehsam, P. Lalezari, R. Lekhraj et al., "Non-thermal radio frequency and static magnetic fields increase rate of hemoglobin deoxygenation in a cell-free preparation," PLOS ONE, vol. 8, no. 4, Article ID e61752, 2013.

[47] D. J. Muehsam and A. A. Pilla, "A Lorentz model for weak magnetic field bioeffects: part I-thermal noise is an essential component of AC/DC effects on bound ion trajectory," Bioelectromagnetics, vol. 30, no. 6, pp. 462-475, 2009.

[48] D. J. Muehsam and A. A. Pilla, "A Lorentz model for weak magnetic field bioeffects: part II-secondary transduction mechanisms and measures of reactivity," Bioelectromagnetics, vol. 30, no. 6, pp. 476-488, 2009. 


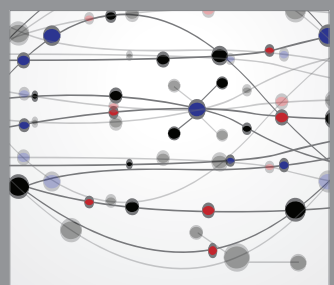

The Scientific World Journal
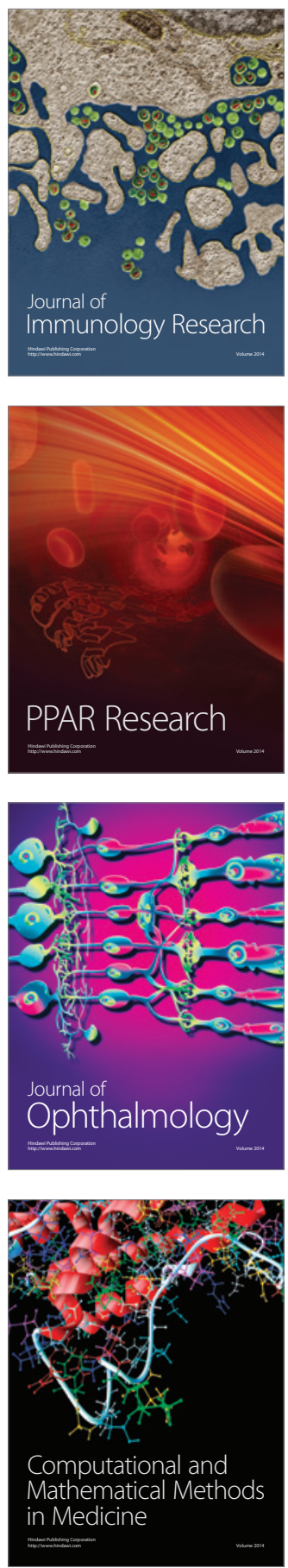

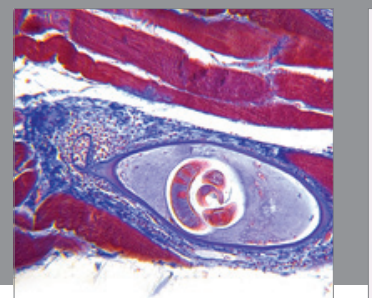

Gastroenterology

Research and Practice
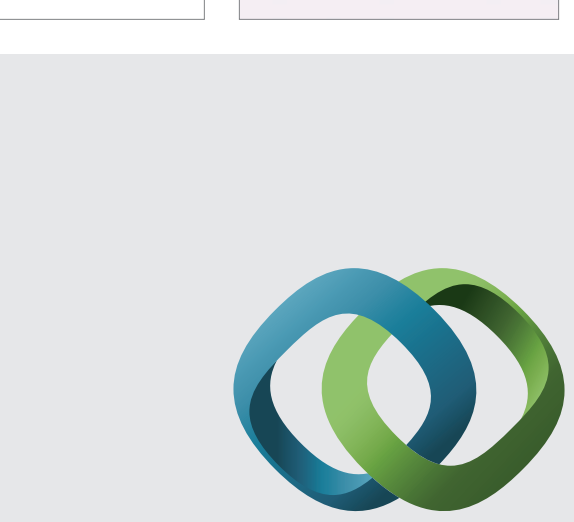

\section{Hindawi}

Submit your manuscripts at

http://www.hindawi.com
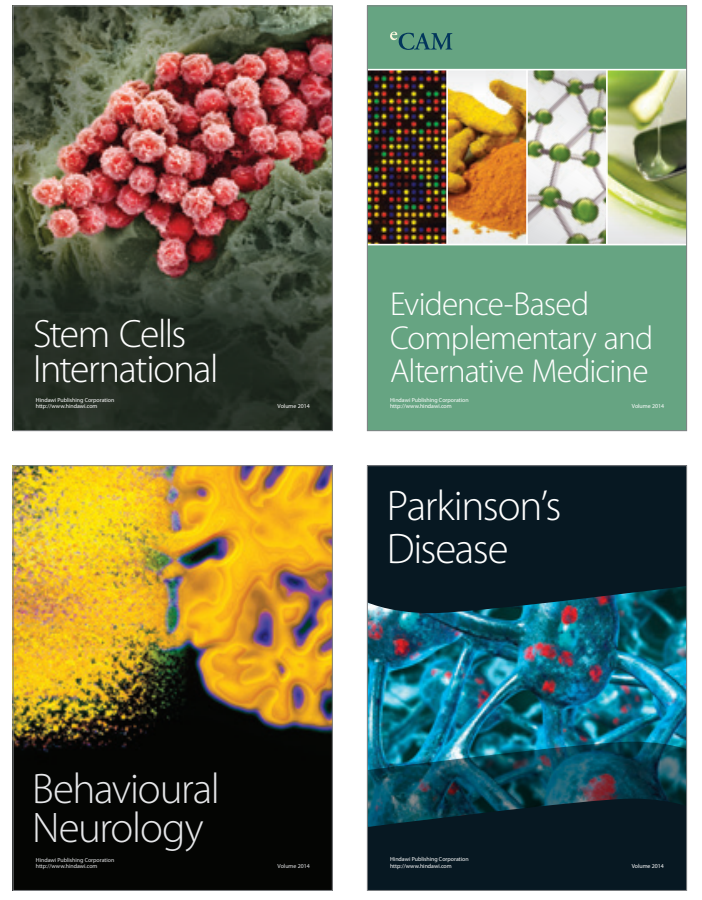
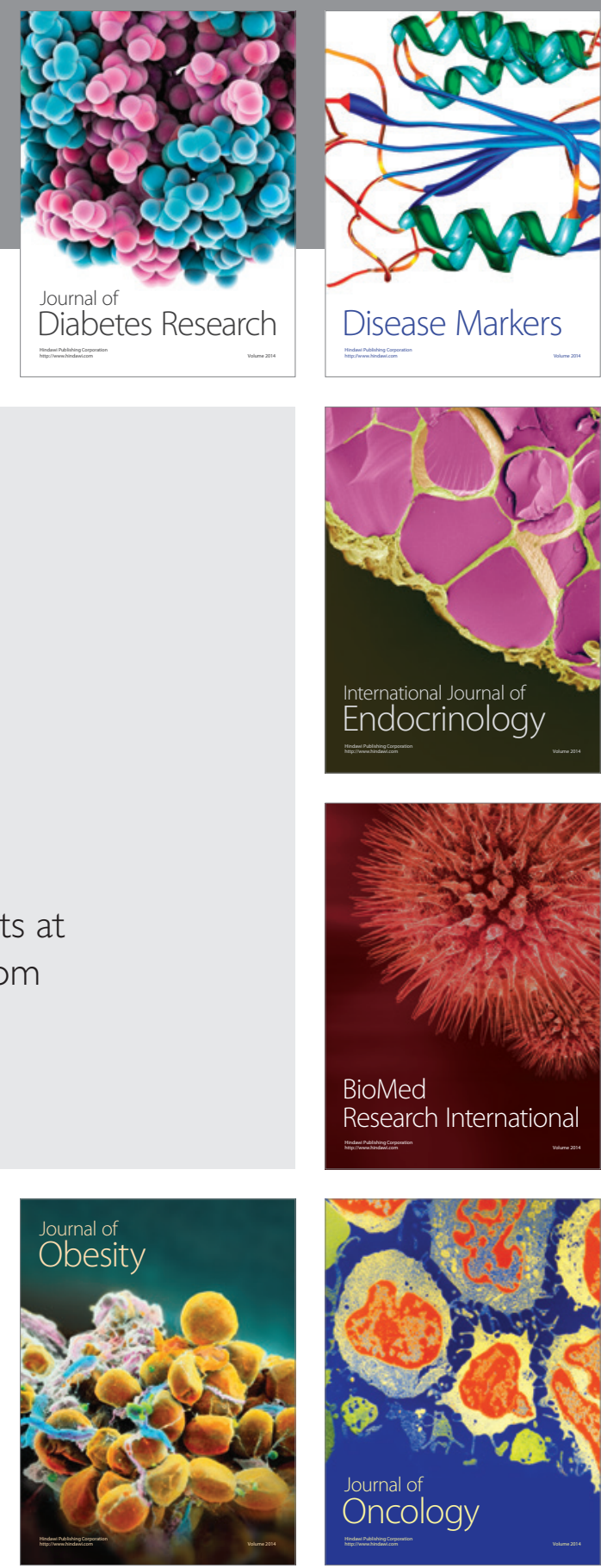

Disease Markers
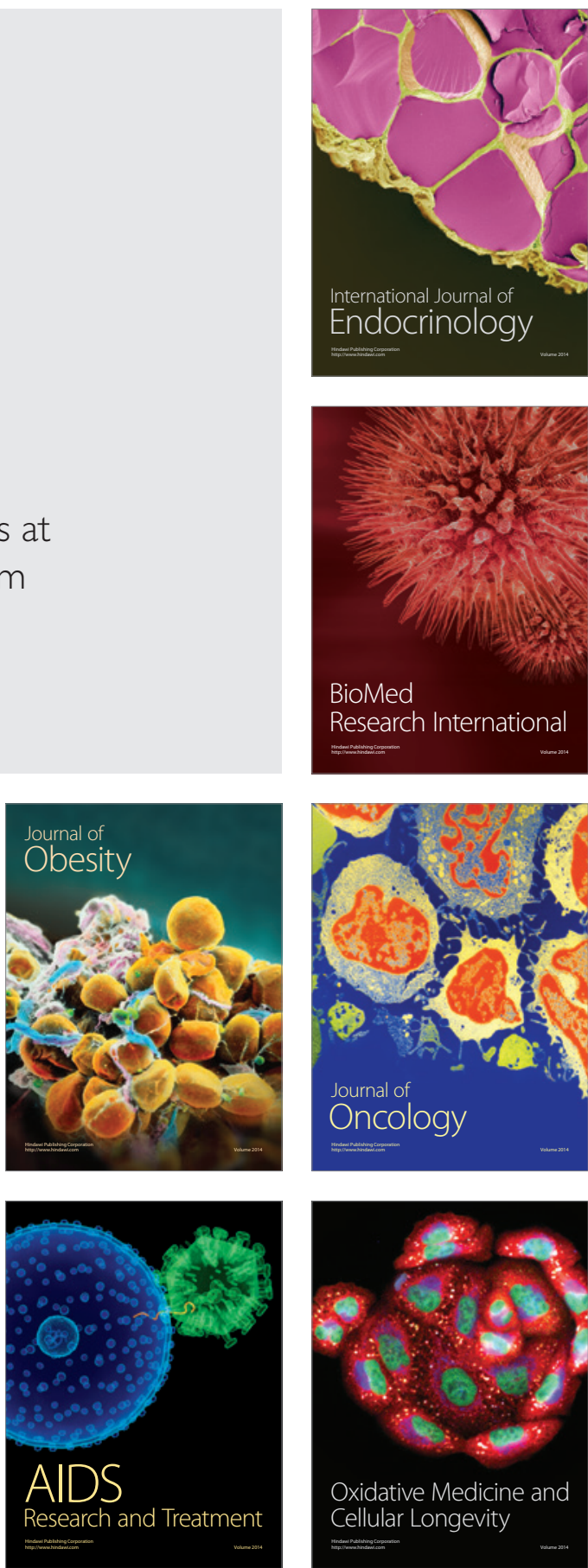